\title{
BMJ Open Advancing Concussion Assessment in Pediatrics (A-CAP): a prospective, concurrent cohort, longitudinal study of mild traumatic brain injury in children: study protocol
}

Keith Owen Yeates, ${ }^{1,2,3}$ Miriam Beauchamp, ${ }^{4}$ William Craig, ${ }^{5}$ Quynh Doan, ${ }^{6}$ Roger Zemek, ${ }^{7}$ Bruce H Bjornson, ${ }^{6}$ Jocelyn Gravel, ${ }^{8}$ Angelo Mikrogianakis, ${ }^{3}$ Bradley Goodyear, ${ }^{2,9}$ Nishard Abdeen, ${ }^{10}$ Christian Beaulieu, ${ }^{11}$ Mathieu Dehaes, ${ }^{12}$ Sylvain Deschenes, ${ }^{12}$ Ashley Harris, ${ }^{2,3,9}$ Catherine Lebel, ${ }^{3,9}$ Ryan Lamont, ${ }^{3,13}$ Tyler Williamson, ${ }^{14}$ Karen Maria Barlow, ${ }^{3,15,16}$ Francois Bernier, ${ }^{3,17}$ Brian L Brooks, ${ }^{3,15,16}$ Carolyn Emery, ${ }^{2,3,18}$ Stephen B Freedman, 3,15 Kristina Kowalski, ${ }^{1,3}$ Kelly Mrklas, ${ }^{19}$ Lianne Tomfohr-Madsen, ${ }^{1,3}$ Kathryn J Schneider, ${ }^{2,3,18}$ on behalf of Pediatric Emergency Research Canada (PERC)

To cite: Yeates KO, Beauchamp M, Craig W, et al. Advancing Concussion Assessment in Pediatrics (A-CAP): a prospective, concurrent cohort, longitudinal study of mild traumatic brain injury in children: study protocol. BMJ Open 2017;7:e017012. doi:10.1136/ bmjopen-2017-017012

- Prepublication history for this paper is available online. To view these files, please visit the journal online (http://dx.doi. org/10.1136/bmjopen-2017017012).

Received 26 March 2017 Revised 5 June 2017 Accepted 6 June 2017

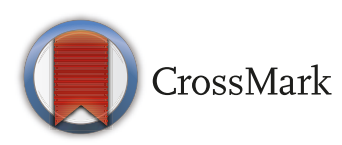

For numbered affiliations see end of article.

Correspondence to Dr. Keith Owen Yeates; kyeates@ucalgary.ca

\section{ABSTRACT}

Introduction Paediatric mild traumatic brain injury (mTBI) is a public health burden. Clinicians urgently need evidence-based guidance to manage $\mathrm{mTBI}$, but gold standards for diagnosing and predicting the outcomes of $\mathrm{mTBl}$ are lacking. The objective of the Advancing Concussion Assessment in Pediatrics (A-CAP) study is to assess a broad pool of neurobiological and psychosocial markers to examine associations with postinjury outcomes in a large sample of children with either mTBI or orthopaedic injury (OI), with the goal of improving the diagnosis and prognostication of outcomes of paediatric mTBI.

Methods and analysis A-CAP is a prospective, longitudinal cohort study of children aged 8.0016.99 years with either $\mathrm{mTBI}$ or $0 \mathrm{O}$, recruited during acute emergency department (ED) visits at five sites from the Pediatric Emergency Research Canada network. Injury information is collected in the ED; follow-up assessments at 10 days and 3 and 6 months postinjury measure a variety of neurobiological and psychosocial markers, covariates/confounders and outcomes. Weekly postconcussive symptom ratings are obtained electronically. Recruitment began in September 2016 and will occur for approximately 24 months. Analyses will test the major hypotheses that neurobiological and psychosocial markers can: (1) differentiate mTBI from $\mathrm{OI}$ and (2) predict outcomes of mTBI. Models initially will focus within domains (eg, genes, imaging biomarkers, psychosocial markers), followed by multivariable modelling across domains. The planned sample size (700 mTBI, 300 0l) provides adequate statistical power and allows for internal cross-validation of some analyses.

\section{Strengths and limitations of this study}

- The study will be the largest of its kind to date, measuring a broad pool of neurobiological and psychosocial markers in 1000 children and assessing their outcomes across the first 6 months postinjury.

- Genetic studies will help identify potential mechanisms underlying neural injury and repair in mild traumatic brain injury (mTBI).

- State-of-the-art neuroimaging will afford a detailed assessment of the neural substrates of mTBI that also can be linked to genetic profiles, as well as to outcomes.

- The inclusion of novel psychosocial markers will yield a better appreciation of non-injury influences on mTBI and how they interact with its neurobiological substrates.

- Not all children with mTBI present to paediatric emergency departments; thus the study's findings may not be generalisable to children presenting to other settings or who do not seek medical care.

- The study will only assess vestibular-ocular and cervicogenic problems indirectly, based on ratings of relevant postconcussive symptoms and pain as well as assessment of balance.

Ethics and dissemination The ethics boards at all participating institutions have approved the study and all participants and their parents will provide informed consent or assent. Dissemination will follow an integrated knowledge translation plan, with study findings presented at scientific conferences and in multiple manuscripts in peer-reviewed journals. 


\section{INTRODUCTION}

Paediatric mild traumatic brain injury (mTBI) is a public health burden. Millions of children in North America sustain mTBI annually, and the numbers seeking care are rising dramatically. ${ }^{12}$ Children with mTBI often report postconcussive symptoms (PCS), including somatic (eg, headache, dizziness), cognitive (eg, inattention, forgetfulness) and affective (eg, irritability, dysphoria) complaints. PCS are most severe acutely but persist for weeks or months in 15\%-30\% of cases and can result in functional disability and declines in quality of life. ${ }^{3}{ }^{4}$ Clinicians urgently need evidence-based guidance to manage mTBI. The Canadian Institutes of Health Research, National Institutes of Health, Centers for Disease Control and Prevention and WHO have all called for more research to inform clinical care and improve outcomes for children with mTBI. ${ }^{5-7}$

A gold standard for diagnosing and predicting the prognosis of mTBI is lacking. Multiple methods are used to assess mTBI (eg, clinical assessment of presenting signs/ symptoms, balance testing, neuropsychological testing, neuroimaging). Each has value for diagnosis and prognosis, and multivariate models that combine multiple measures have shown utility both in differentiating mTBI and in forecasting its outcomes. ${ }^{89}$ However, these models thus far lack the precision needed to direct clinical care of individual patients. Psychosocial markers (eg, ineffective coping skills, parental distress) help to predict persistent PCS and related functional impairments, over and above neurobiological markers. ${ }^{10}$ Thus, a multidimensional approach that incorporates both neurobiological and psychosocial markers is needed for effective diagnosis and prognosis, as well as to inform treatment strategies. ${ }^{11}$ However, no study to date has had a sample large enough to systemically examine the broad pool of markers and their likely interactions for the purposes of either diagnosis or prognosis.

Innovative research is therefore needed to improve the assessment of mTBI. Previous studies have been hampered by a variety of shortcomings. Many had sample sizes too small to examine complex interactions involving the broad pool of diagnostic and prognostic markers. Other large studies did not include several promising neurobiological and psychosocial markers. ${ }^{12} 13$ For instance, multiple genes are implicated in response to neurotrauma, neural repair and plasticity, and cognitive reserve and thus are likely to affect the expression of mTBI. ${ }^{14}$ Single genes have been studied in isolation, but a fuller complement of genes has not been examined in any previous study. Neuroimaging studies have generally focused on a single modality in small samples and are often underpowered statistically. Finally, psychosocial markers known to be critical for recovery from injuries and trauma in general (eg, psychological resilience) have not been studied in the context of mTBI. ${ }^{1516}$

To make significant advances in the scientific understanding of mTBI and its clinical management, larger, multisite observational studies are needed that incorporate several key elements: prospective designs; large samples drawn from representative populations; rigorous definitions of $\mathrm{mTBI}$; appropriate comparison groups; comprehensive measurement of neurobiological and psychosocial markers, as well as a range of outcomes; and consideration of potential confounders, covariates and moderators. No study to date has embodied all of these elements.

\section{Objectives}

The broad objective of the Advancing Concussion Assessment in Pediatrics (A-CAP) study is to improve the diagnosis and prognosis of paediatric mTBI. The aim is to conduct a multisite, prospective, concurrent cohort study that will assess a broad pool of neurobiological and psychosocial markers, including some not yet examined in any large-scale study (eg, multiple candidate genes, psychological resilience), and assess outcomes longitudinally over the first 6 months postinjury. The study will have a large sample to enable the analysis of complex interactions among neurobiological and psychosocial markers in terms of both diagnosis and prognosis of outcomes. We hypothesise that this strategy will (1) identify mTBI with excellent sensitivity and specificity (ie, differentiate it from orthopaedic injury) and (2) accurately predict longitudinal outcomes of mTBI. The research will enhance scientific understanding of paediatric mTBI. Genetic studies will help identify potential mechanisms underlying neural injury and repair in mTBI. State-ofthe-art neuroimaging will afford a detailed assessment of the neural substrates of mTBI that can be linked to genetic profiles, as well as to outcomes. Thus, the study will help identify biomarkers for mTBI and clarify their prognostic significance. Similarly, the inclusion of novel psychosocial markers will yield a better appreciation of non-injury influences on mTBI and how they interact with its neurobiological substrates.

\section{METHODS \\ Study overview}

The study uses a prospective longitudinal cohort design involving children aged 8.00 to 16.99 years who have either an mTBI or an orthopaedic injury (OI). Children with OI were chosen as a comparison group because they are comparable to children with mTBI demographically and in terms of background characteristics that may affect outcomes, ${ }^{17}$ and also because they share a common exposure to a traumatic injury; thus, their inclusion helps to control for the general effects of trauma on symptom reporting and the non-specific nature of PCS. Recruitment occurs during acute emergency department (ED) visits at five sites, all of which are members of the Pediatric Emergency Research Canada (PERC) network: Alberta Children's Hospital (Calgary), Children's Hospital of Eastern Ontario (CHEO; Ottawa), Ste Justine Hospital (Montreal), Stollery Children's Hospital (Edmonton) and British Columbia Children's Hospital (Vancouver). The 
inclusion of five sites across Canada ensures a representative sample that is sufficiently large (700 mTBI, $300 \mathrm{OI})$ to provide adequate statistical power given the anticipated number of diagnostic/prognostic markers and their estimated effect sizes, and also to permit splitting the sample in half when conducting more discovery-based analyses and cross-validating results in the remaining participants. Injury information is collected in the ED. Follow-up assessments conducted within 10 days postinjury and at 3 and 6 months postinjury measure a broad pool of neurobiological and psychosocial markers, potential covariates and confounders, and outcomes (ie, PCS, functional disability, quality of life). Weekly PCS ratings are obtained electronically via smartphones or the internet. Most measures are drawn from National Institutes of Health (NIH) and Canadian Institutes of Health Research Common Data Elements for mTBI. ${ }^{18}$ Recruitment began in September 2016 and will occur for approximately 24 months, with another 6 months needed to complete all follow-up visits; thus the study should conclude by mid-2019.

\section{Study population}

Participants range from 8.00 to 16.99 years of age at the time of their injuries. The exclusion of younger children helps to insure that participants will be capable of self-report and able to complete MRI without sedation. Older adolescents are excluded in part for practical reasons, because they appear relatively infrequently in the EDs at the participating sites, but also to reduce developmental heterogeneity within the sample; we wanted the study to focus on school-aged children and youth, not on young adults, who function in very different contexts from their younger counterparts.

\section{Inclusion criteria}

The mTBI group includes children who sustain a blunt head trauma resulting in one or more of the following three criteria, consistent with the WHO definition of mTBI: (1) an observed loss of consciousness, (2) a Glasgow Coma Scale score of 13 or 14 or (3) at least one acute sign or symptom of concussion as noted by ED medical personnel on a standard case report form (ie, post-traumatic amnesia, focal neurological deficits, skull fracture, post-traumatic seizure, vomiting, headache, dizziness, other mental status changes). Children with OI are included if they sustain upper or lower extremity fractures, sprains or strains due to blunt force/physical trauma, associated with Abbreviated Injury Scale (AIS) ${ }^{19}$ scores of 4 or less. Children in both groups must present to one of the five EDs within 48 hours of injury; transfer from an outside hospital is not an exclusion criterion as long as enrolment occurs within 48 hours of injury.

\section{Exclusion criteria}

Children with mTBI are excluded if they demonstrate delayed neurological deterioration (ie, any Glasgow Coma Scale $(\mathrm{GCS})<13$ ) or require neurosurgical intervention. Children whose injuries are accompanied by a loss of consciousness for more than 30 min or post-traumatic amnesia greater than 24 hours are excluded, as are those with any associated injury with an AIS scores greater than 4, although they may have associated injuries of lesser severity. Exclusion criteria for children with OI include any head trauma or symptoms of concussion, as well as any injury requiring surgical intervention or procedural sedation; this includes closed reductions. Children are not excluded from either group if they are administered analgesic medication, including narcotics if used solely for pain management. The administration of pain medication is tracked and will be treated as a covariate in data analyses. Children who wear braces/spacers or have other metal in the mouth, or who have medical contraindications to MRI, are only excluded from the MRI portion of the study.

Additional exclusion criteria for both groups are as follows: (1) hypoxia, hypotension or shock during or following the injury (if known at the time of recruitment); (2) non-English-speaking child or parents (non-English and non-French-speaking in Quebec or Ottawa); (3) previous TBI requiring overnight hospitalisation, by parent report; (4) previous concussion within the past 3 months, by parent report, to exclude children still in the acute phase of recovery; (5) previous neurological or neurodevelopmental disorder such as epilepsy, intellectual disability/mental retardation, autism, by parent report (history of attention deficit hyperactivity disorder, learning disability or Tourette's syndrome is not an exclusion); (6) hospitalisation in the previous year for psychiatric disorder, by parent report; (7) administration of sedative medication (propofol, ketamine, nitrous oxide, midazolam, benzodiazepines) prior to ED data collection (fentanyl is not an exclusion if used for pain management only); (8) obvious alcohol or drug ingestion associated with injury; (9) injury related to abuse or assault and (10) legal guardian not present or child in foster care.

\section{Study procedures}

\section{Recruitment}

Recruitment occurs in the EDs at each of the five sites by dedicated staff that complete screening of all potentially eligible children who present to the ED during study enrolment hours (approximately 08:00-23:00, 7 days a week, depending on available staffing). Eligible and willing parents, along with adolescents capable of consenting on their own behalf, are asked for written informed consent; assent is requested from children who are not capable of providing informed consent themselves. Physicians are notified of enrolment and asked to complete a standardised case report form regarding the participant's medical presentation.

\section{Data collection}

At the time of recruitment, research staff members collect information regarding the injury itself and assess children's 
acute clinical presentation, balance and neurocognitive function. After initial data collection is completed in the $\mathrm{ED}$, the local research coordinator contacts families to set up initial postinjury follow-up assessments that occur as soon as possible within 10 days postinjury. The children and their families are then assessed postacutely (within 10 days post-injury) and again at 3 - and 6 months post-injury.

In addition to the ED and follow-up assessments, parents and children provide ratings of postconcussive symptoms and pain, weekly for the first 3 months and biweekly from 3 to 6 months, using a mobile phone application or web-based survey, depending on their preference. Both child and parent complete independent surveys, either on a shared mobile phone or separately if each has their own device. The mobile phone application and internet surveys function in a similar fashion, with a reminder prompt or email sent weekly. Participants have a 72 hour window to complete each survey. Once a survey is completed, the answers are no longer visible on the mobile device or internet. Data are uploaded automatically. Surveys on the phone application can be completed offline, and data are uploaded automatically once the device is back online.

The postacute assessment (within 10 days) includes measures of neurobiological and psychosocial markers, postconcussive symptoms and functional impairments, and potential covariates, confounders and moderators. Children provide a saliva sample to obtain their DNA for genetic analyses and complete MRI scanning. Subsequent follow-up assessments at 3 and 6 months postinjury include similar measures used at the postacute assessment (eg, symptoms and postinjury functional impairments); intellectual functioning is measured only at 3 months, because it is not routinely altered by mTBI. At either the 3-month or 6-month visit, children complete a second MRI; this is determined using a random number table at the time of the postacute assessment. At all visits, we also collect demographic information, as well as information about subsequent injuries and any interventions and treatments received, including educational services.

The postacute assessment lasts about 2 hours, with an additional hour for MRI, and the 3 and 6-month assessments last approximately $90 \mathrm{~min}$. Completion of ratings at home takes about 5 min per week each for parent and child. Parents and children are reimbursed for their time during each visit with gift certificates. A small incentive for each completed symptom rating is offered to the child to encourage regular participation. Multiple methods are used to track participants and reduce attrition, which should be $10 \%-15 \%$ based on previous studies by the lead investigator and his collaborators. ${ }^{10} 172021$

\section{Measures}

Table 1 summarises the measures used in the study. All measures have demonstrated satisfactory reliability (ie, internal consistency, inter-rater agreement and/or testretest reliability) and validity.

\section{Diagnostic/prognostic markers}

Acute signs and symptoms

Information about children's injuries is collected from medical records and medical personnel using a standardised case report form to elicit details regarding the injury and acute signs and symptoms of concussion (ie, loss of consciousness, GCS scores, mechanism of injury, neurological status, reports of retrograde or anterograde amnesia, emesis and other clinical features). The variables of interest were selected based on large-scale studies of the risk factors associated with an increased/decreased risk of significant intracranial injury after $\mathrm{mTBI},{ }^{22-25}$ as well as based on the results of the recent PERC Predicting Persistent Postconcussive Problems in Pediatrics (5P) study. ${ }^{9}$ Information is collected by recruiters and verified by attending physicians, a process shown in previous research to yield reliable data. ${ }^{25}$

\section{Candidate genes}

Multiple genes are implicated in response to neurotrauma, neural repair and plasticity, and cognitive reserve. ${ }^{14}$ Although single genes have been studied in isolation, ${ }^{26}$ a fuller complement of genes has not been examined in any previous large-scale study. Candidate genes and single-nucleotide polymorphisms (SNPs) were identified based on their involvement in neurotrauma response (eg, interleukin 1 alpha [IL1A]), neural repair and plasticity (eg, brain-derived neurotrophic factor [BDNF], apolipoprotein $\mathrm{E}[\mathrm{APOE}])$, and cognitive reserve (eg, solute carrier family 6 member 3[SLC6A3, previously known as DAT]). ${ }^{14}$ Saliva samples are collected using the Oragene DNA OG-500 self-collection kit. Samples are labelled by study identification only and sent to the Calgary site for processing. Sputum is separated from saliva and dispersed using dithiothreitol. The dispersed suspension is then centrifuged and filtered. Genetic analyses will be conducted by a central laboratory at Calgary by technicians blinded to exposure group and outcome.

\section{Magnetic resonance imaging}

All children (both mTBI and OI) without any contraindications to MRI complete imaging at the postacute assessment and are randomised to complete a second MRI at either the 3-month or 6-month follow-up assessment. A second scan is being obtained in part to determine if differences in brain structure or function can be detected following symptom resolution or only occurs in concert with persistent symptoms. Each brain scanning session lasts 45-60 min and includes the following sequences: three-dimensional T1-weighted volumetric - to measure regional volumes and cortical thickness; diffusion tensor imaging-to assess the integrity of white matter microstructure; quantitative susceptibility mapping-to assess haemorrhage and iron deposition; resting state functional MRI-to assess functional connectivity; T2-weighted fluid attenuated inversion recovery, for lesion detection; arterial spin labelling-to assess perfusion; and proton spectroscopy in a single voxel in the dorsolateral 


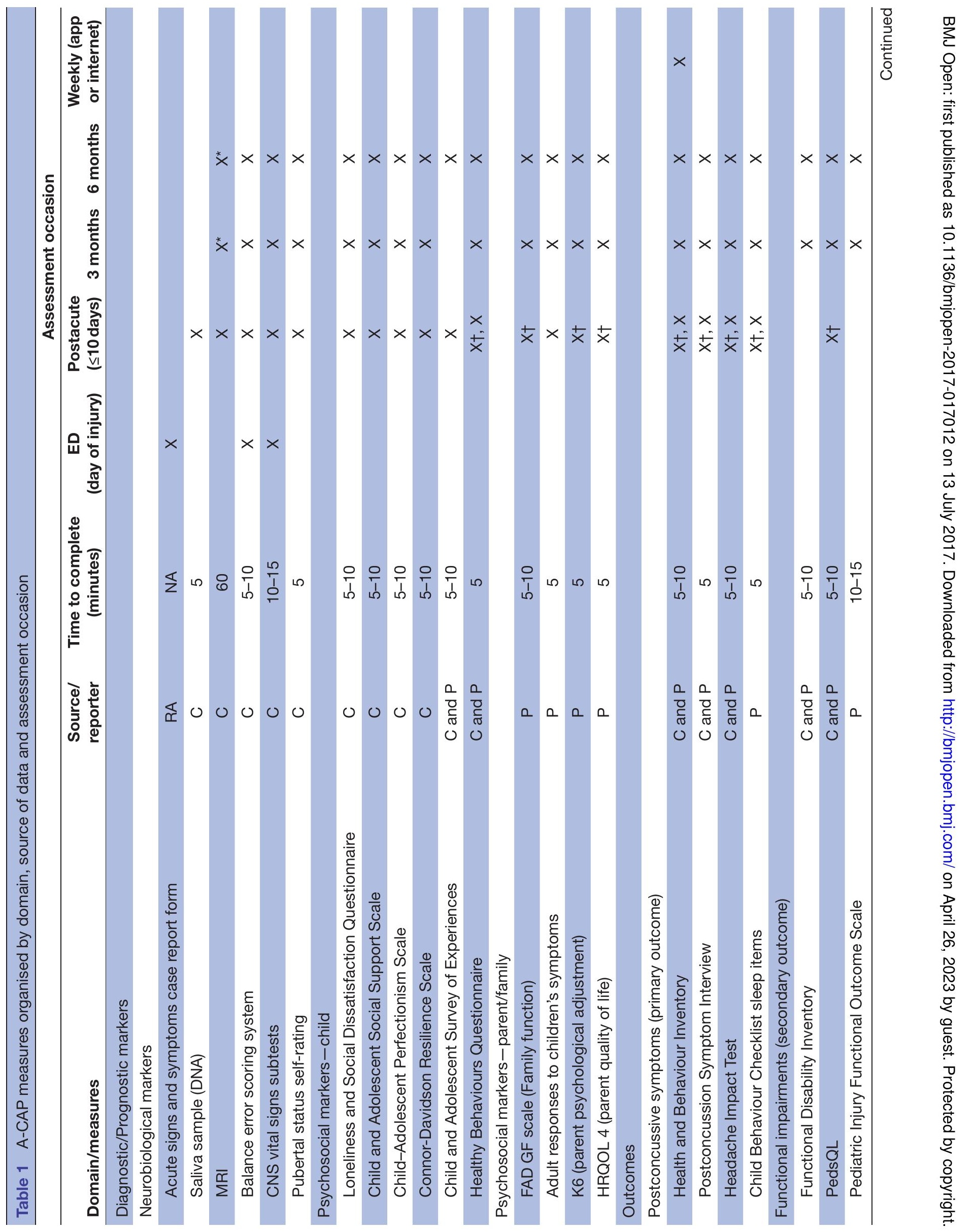




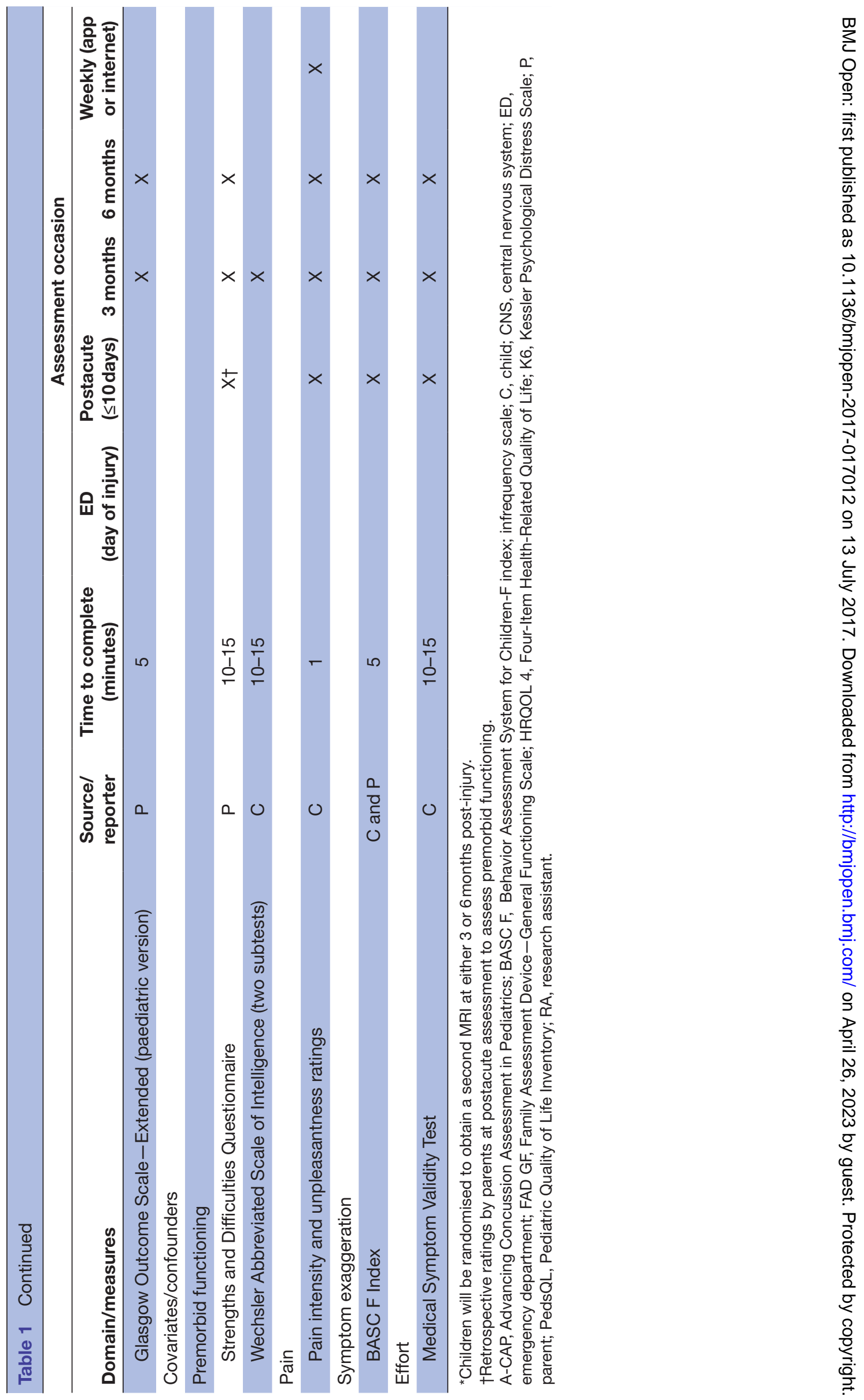


prefrontal cortex-to assess metabolite concentrations. All imaging takes place using a single 3T scanner at each site (General Electric MR750w in Calgary; General Electric MR750 in Montreal and Vancouver; Siemens Prisma in Edmonton; Siemens Skyra in Ottawa). Table 2 summarises the key imaging parameters for the two scanner types. Foam padding is used to minimise head motion and headphones are provided to minimise noise. Participants are not sedated.

\section{Balance}

Balance is assessed using the Balance Error Scoring System (BESS), ${ }^{27}$ which already has wide application in the field of sports concussion ${ }^{28}$ and was used in the $5 \mathrm{P}$ study. Two stances are used: narrow double-leg stance and tandem stance (hard surface only). Each stance is held, with hands on hips and eyes closed, for $20 \mathrm{~s}$. Error points are given for specific behaviours, including opening eyes, lifting hands off hips or stepping, stumbling or falling. Significant correlations between the BESS and force platform sway measures have been established with healthy individuals. ${ }^{29}$ The BESS has shown satisfactory reliability in children and adolescents. ${ }^{30}$ Between $10 \%$ and $20 \%$ of the assessments will be double scored to check on the reliability of scoring.

\section{Neuropsychological function}

Neurocognitive skills, including processing speed, executive functioning and visual memory, are assessed using three of the seven subtests of the computerised Central Nervous System Vital Signs test battery: the Stroop Test, Shifting Attention Test and Visual Memory Test. These three subtests can be completed in about $15 \mathrm{~min}$ with minimal disruption to the ED flow, ${ }^{31}$ and have been shown to discriminate children with concussion from children with OI, ${ }^{32}$ to predict symptoms 1 month after concussion ${ }^{33}$ and to not negatively alter symptom recovery. ${ }^{34}$ The tests generate composite scores for reaction time, cognitive flexibility and visual memory.

\section{Pubertal status}

Pubertal status is assessed using a self-administered rating scale. The scale has separate questions for boys and girls, and asks them to rate changes to their body on a four-point Likert-type Scale. Point values are averaged to produce a Pubertal Development Score,${ }^{35}$ which shows adequate agreement with direct clinical assessment of Tanner staging. The scale avoids the use of pictorial diagrams, which could be objectionable to children or their parents, and is therefore more appropriate in the context of this study.

\section{Loneliness}

The Loneliness and Social Dissatisfaction Questionnaire-Short Version is a nine-item rating scale on which respondents answer on a 5-point Likert-type scale. The scale focuses on feelings of loneliness among children and adolescents and produces a total loneliness score, with higher scores reflecting higher levels of loneliness. ${ }^{36}$
This is a short form version of the widely used Loneliness and Social Dissatisfaction Questionnaire. ${ }^{37}$

\section{Social support}

The Child and Adolescent Social Support Scale is a 40-item scale that measures perceived social support from four sources: parents, teachers, classmates and friends. It asks children to respond to statements such as, 'My parent(s) help me make decisions', by rating frequency on a 6-point Likert scale. Subscale scores are computed by summing frequency scores for each source of support, and a total score is calculated by summing all four frequency subscale scores. ${ }^{38}$

\section{Perfectionism}

The Child-Adolescent Perfectionism Scale is a 22-item rating scale on which respondents answer using a 5-point Likert-type scale. The scale measures three different aspects of perfectionism, including self-oriented striving perfectionism, self-oriented critical perfectionism and socially prescribed perfectionism. Higher scores on the scale are associated with psychological distress and maladjustment in children and adolescents. ${ }^{39}$

\section{Resilience}

The Connor-Davidson Resilience Scale 10 is a 10 -item rating scale designed to measure people's perception of their own psychological resilience. Each item is rated on a 5-point Likert scale. The scale is summed and provides a total score (0-40) for the overall level of resilience (lower scores suggest lesser levels of resilience). ${ }^{40}$ Scores have been shown to be associated with postconcussive symptoms in adults with mTBI. ${ }^{41}$

\section{Life events}

The Child and Adolescent Survey of Experiences (CASE) requires respondents to indicate whether 38 listed life events have occurred in the previous 12 months and to rate reported events as either good or bad. The scale generates total scores for good and bad life events. Reports on the CASE have been shown to correlate with children's psychosocial adjustment. ${ }^{42}$

\section{Healthy behaviours}

Children's mental and physical activity (including rest), diet, sleep and screen time is assessed using the Healthy Lifestyle Behaviours Questionnaire (HBLQ), which includes 26 items on which respondents report the frequency of each behaviour over the past week. Items for the HLBQ are drawn from existing validated measures of health behaviour in children and youth (eg, Health Behaviour of School Aged Children Study; Adolescent Sleep Hygiene Scale) ${ }^{43}{ }^{44}$ Retrospective ratings of preinjury behaviours will be obtained at the postacute assessment, and ratings of postinjury behaviours will be obtained at all postinjury assessments. 


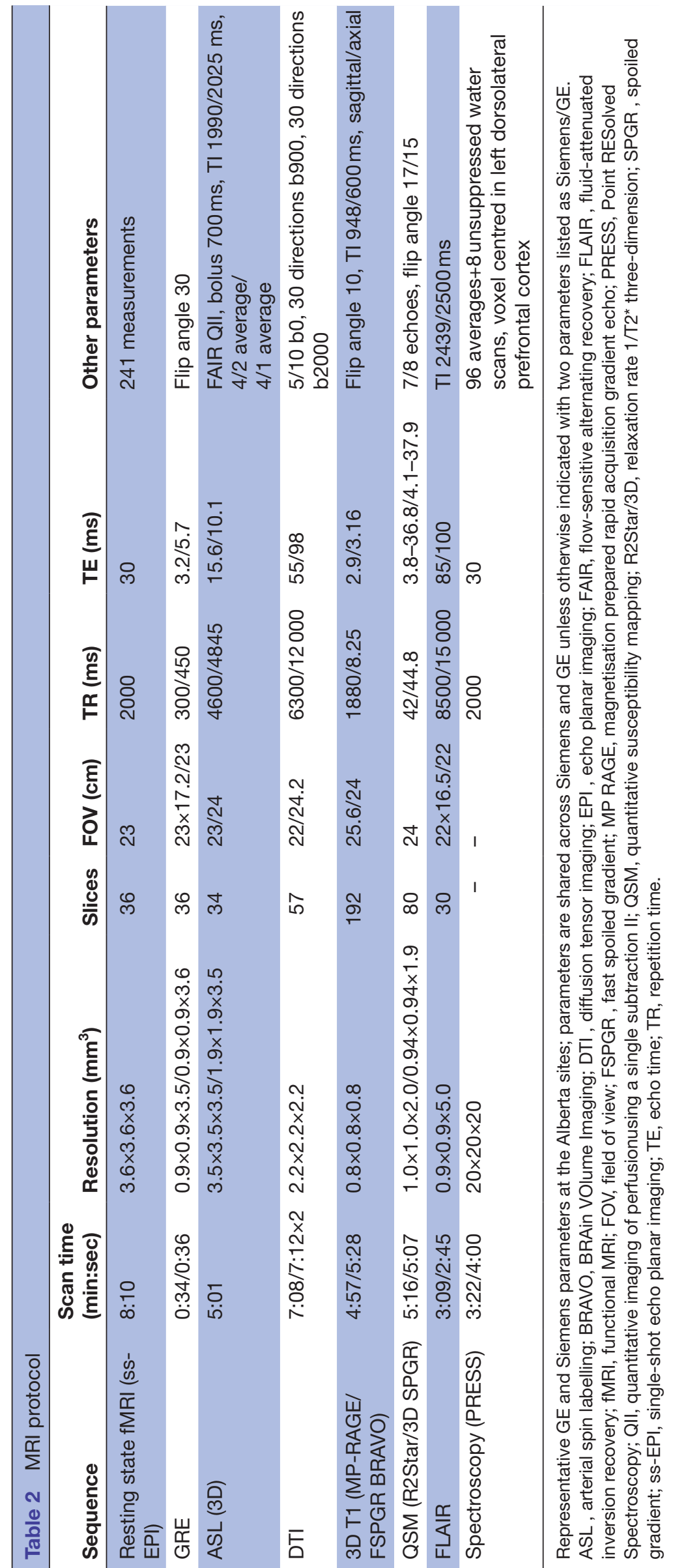




\section{Family function}

Family adjustment is assessed by obtaining ratings from parents on the 12-item General Functioning scale from the McMaster Family Assessment Device. ${ }^{45} 46$ The measure has shown satisfactory reliability and validity in previous research. Consistent with the scaling of the original scores, higher scores reflect worse family functioning. Better premorbid family functioning has been associated with more pronounced somatic symptoms in children with mTBI, suggesting that children from higher functioning families may be more sensitive to the effects of mTBI. ${ }^{47}$

\section{Response to symptoms}

The Adult Responses to Children's Symptoms scale was developed to assess parent behaviour in the context of children's symptoms. The scale is composed of 29 items rated on a 5-point Likert-type scale. The scale generates four subscales: protect, minimise, monitor and distract (plus solicitousness for teens). Scores on the scales are responsive to intervention and predictive of parental emotional functioning and children's symptoms. ${ }^{48}$

\section{Parent psychological adjustment}

The K6 scale is a six-item rating scale designed to assess non-specific distress and to discriminate cases of serious mental illness from non-cases. ${ }^{49}$ The K6 was developed with support from the US government's National Center for Health Statistics for use in the redesigned US National Health Interview Survey (NHIS). The K6 is included in the core of the NHIS, as well as in the annual National Household Survey on Drug Abuse. ${ }^{50}$

\section{Parent quality of life}

Parent quality of life is measured using the four-item set of Healthy Days core questions from the US Department of Health and Human Services' Centers for Disease Control and Prevention (CDC). The CDC Four-Item Health-Related Quality of Life includes a self-rated measure of general health, as well as a report of the number of recent days that an individual has been physically unhealthy, mentally unhealthy and limited in regular activity. ${ }^{51}$

\section{Outcome measures}

Postconcussive symptoms

Ratings of postconcussive symptoms are obtained using the Health and Behaviour Inventory (HBI) ${ }^{52}$ and the Post-Concussive Symptom Interview (PCS-I). ${ }^{53}$ The measures differ in administration format (written vs oral), response format (Likert-style rating vs yes/no) and scoring (symptom frequency vs symptom count) and therefore provide overlapping but distinct types of information. Both measures have child self-rating and parent proxy forms. The HBI has been adopted as a core measure in the Common Data Elements (CDE) for Pediatric Traumatic Brain Injury. ${ }^{18}$ It yields separate scores for cognitive and somatic symptom scales. The PCS-I is a structured oral interview that asks about the presence of symptoms similar to those listed for postconcussional disorder in Diagnostic and Statistical Manual of Mental Disorders 4th Edition.

A more specific measure of headache is obtained because it is one of two PCS, along with dizziness, that has previously been shown to best differentiate children with mTBI from those with OI. ${ }^{54}$ The Headache Impact Test (HIT-6) provides a global measure of adverse headache impact. Its items measure the adverse impact of headache on social functioning, role functioning, vitality, cognitive functioning and psychological distress. The HIT-6 also measures the severity of headache pain. It is included in the Canadian mTBI CDE. ${ }^{55}$

Because of concerns about the effects of mTBI on sleep and an increased risk of sleep disturbance, the children and their parents also complete ratings on the seven sleep items from the Child Behaviour Checklist (CBCL). The CBCL is a widely used measure of emotional and behavioural adjustment; the sleep items correlate significantly with other validated instruments designed specifically to assess sleep. ${ }^{56}$

\section{Functional impairments}

Children and parents will complete the Functional Disability Inventory (FDI $)^{57} 58$ to assess activity limitations attributable to their injuries. The FDI elicits ratings concerning perceptions of activity limitations due to physical health problems. Parents will also report on functional outcomes by completing the Pediatric Injury Functional Outcome Scale (PIFOS). The PIFOS is a structured interview of 26 items that elicits ratings regarding motor skills, daily living skills, communication skills, cognition, socialemotional functioning, physical changes and academic functioning. ${ }^{59}$

The Pediatric Quality of Life Inventory (PedsQL) ${ }^{6061}$ will be administered to parents and children to assess broader aspects of children's quality of life. The PedsQL measures children's health in terms of physical, emotional, social and school functioning. The four subscales generate psychosocial and physical summary scores, as well as a total health score. Global functional outcomes will be assessed using the paediatric version of the Glasgow Outcome Scale-Extended (GOS-E), ${ }^{62} 63$ which is the core measure of global outcome in the NIH Common Data Elements. The GOS-E is completed based on a brief structured interview.

\section{Covariates/confounders/moderators}

We will collect information from participants and their parents about a variety of potential covariates, confounders and moderators, including age, sex, previous concussion history, history of migraine headaches and treatments received for their injuries. In addition, participants and their parents will complete a variety of relevant measures.

\section{Premorbid psychosocial adjustment}

The Strength and Difficulties Questionnaire (SDQ) ${ }^{64}$ is a reliable and valid standardised parent questionnaire assessing children's emotional symptoms, conduct 
problems, hyperactivity/inattention, peer relationships and prosocial behaviour. It takes approximately $10 \mathrm{~min}$ to complete. Parents complete the SDQ retrospectively at the postacute assessment to assess children's premorbid psychosocial adjustment. They complete the SDQ at the 3-month and 6-month assessments to measure children's postinjury psychosocial adjustment.

\section{Intellectual functioning}

Children's general intellectual functioning is assessed at the 3-month assessment using the two-subtest version of the Wechsler Abbreviated Scale of Intelligence-Second Edition (WASI-II). ${ }^{65}$

\section{Pain}

Pain intensity is assessed by asking children to rate the severity of the pain they have experienced over the previous week using an 11-point numeric scale, with anchors of 0 (no pain) to 10 (worst pain possible) ${ }^{66}$ Children are also asked to rate pain unpleasantness by asking them to rate how much they are bothered or upset by their pain using a 5-point Likert scale.

\section{Symptom exaggeration}

Symptom exaggeration is assessed using the F Index from the Behavior Assessment System for Children Second Edition. ${ }^{67}$ The F Index assesses the possibility that respondents provide ratings that are inordinately negative, and is considered a 'fake bad' scale.

\section{Effort}

Children's effort is measured using the Medical Symptom Validity Test (MSVT) ${ }^{68}$ which is a computerised, forcedchoice test designed to detect low effort. It presents 10-word pairs twice on the computer screen. Children are then asked to choose the correct word from pairs consisting of a target and foil, both immediately and after a delay. Children are also asked to recall the words during paired-associate and free recall conditions. Children 8 years and older are able to perform at very high levels of recognition accuracy, commensurate with adults. Performance on the MSVT accounts for substantial variance in cognitive test performance among children with mTBI. ${ }^{69} 70$

\section{Data management}

Research Electronic Data Capture (REDCap), ${ }^{71}$ a web-based platform that allows remote data entry from each site, is used to provide common forms for data acquisition, a common database for data entry and common rules for data handling, to insure that data management activities are standardised across sites. All data are entered directly into REDCap whenever possible. Data that are transcribed (eg, intelligence test scores) into REDCap are checked using traditional procedures for double verification, range and error checks and edit trails. Screen entry programmes provide automated calculation of computed variables and perform automated range checks. Data from all sites are merged in a shared central REDCap database stored at the Calgary site.

All MRI data are transmitted via a secure electronic pipeline to the Calgary site for future analysis, managed and curated by the Calgary Image Processing and Analysis Centre (CIPAC; http://www.calgaryimageanalysis.ca/).

\section{Data reduction}

Most of the measures in table 1 were selected in part because they provide composite or summary indices that can be used to represent their specific measurement constructs (eg, the HBI provides summary measures of cognitive and somatic symptoms). Thus, composite or summary variables are available for most of the domains and measures listed in table 1, lessening the need for substantial data reduction procedures.

\section{Missing data}

Multiple imputation will be used when possible to impute missing data if appropriate tests indicate the data are missing at random. ${ }^{72}$ Additionally, statistical techniques such as mixed models and random-effects logistic regression will be used that estimate models based on all available data using maximum likelihood estimation. ${ }^{73} 74$

\section{Data analyses}

Statistical methods

Analyses will evaluate the study's overarching hypotheses that neurobiological and psychosocial markers can: (1) differentiate mTBI from OI and (2) predict outcomes of mTBI. The primary outcome will be postconcussive symptoms as rated on the HBI and PCS-I. Secondary outcomes will include specific measures of headache and sleep (HIT-6, CBCL sleep items), as well as measures of functional outcomes (FDI, PedsQL, PIFOS, GOS-E). Analyses will generally be intended to test a priori hypotheses (eg, we predict a variety of differences in MRI metrics between children with concussion whose symptoms resolve within 4 weeks and those whose symptoms persist beyond 4 weeks) but will be balanced by some discovery-based analyses. One benefit afforded by the large sample size is that internal cross-validation methods can be used for discovery-based analyses.

Models initially will focus within domains (eg, genes, imaging biomarkers, psychosocial markers), followed by multivariable modelling across domains (including tests of interactions among predictors), controlling for multiple comparisons. The analyses will employ a mixed-effects modelling strategy for longitudinal data. Both continuous and dichotomous outcomes will be examined, although the sample size is motivated by the continuous outcomes, particularly postconcussive symptom ratings on the HBI. These methods are robust to departures from normality in the underlying distribution of the outcome; nevertheless, parametric transformations will be considered when distributions are seriously skewed or otherwise fail to meet the assumptions of analysis. Distribution-free alternatives, including rank transformation procedures, will 
be used in cases where parametric transformations are inappropriate.

\section{Diagnostic analyses}

The first central aim of the study is to examine differences between the mTBI and OI groups on both neurobiological and psychosocial markers. Statistical analyses of group differences will be conducted to determine which measures are most sensitive to group differences (ie, able to differentiate children with mTBI from those with OI). Group comparisons will focus on the major groups of neurobiological and psychosocial risk factors (eg, genetic variations and neuroimaging parameters in the neurobiological domain), first independently and then jointly. Differences on some risk factors (eg, genetic variations) may indicate greater vulnerability to concussion, while differences on others (eg, MRI metrics) are likely to be more diagnostic (eg, neuroimaging). The ability of the various measures to discriminate between the mTBI and OI groups will be evaluated using mixed effects modelling, as well as through the use of other traditional indices of clinical utility (eg, sensitivity, specificity, positive and negative predictive values, likelihood ratios and area under the curve in receiver operating curve analyses).

\section{Prognostic analyses}

The second central aim of the research is to examine factors that predict the trajectory of PCS and other outcomes. Specifically, we will investigate the predictive utility of both neurobiological and psychosocial risk factors following mTBI. The major groups of risk factors within those two broad domains (eg, child risk factors within the psychosocial domain) will be investigated independently and jointly in the prediction of PCS. The predictive utility of the various risk factors will be evaluated using mixed-effects modelling, as well as through the use of other traditional indices of clinical utility mentioned above. The modelling strategy will adopt a two-stage approach. In stage one, we will build predictive models for each major class of risk factors individually; then, in stage two, we will examine prediction across risk factors, taking into account possible interactions among predictors. PCS will be treated both as a continuous variable, to maximise power, and as a dichotomy, distinguishing children with persistent increased symptoms from those who do not show persistent symptoms. Recent studies suggest that approximately $30 \%$ of children remain symptomatic by this definition at 4 weeks and about $10 \%-15 \%$ at 3 months. ${ }^{3}{ }^{9}$ The low proportion of children who remain symptomatic at later times post-injury is one reason the study was designed with a large sample size.

In both diagnostic and prognostic analyses, several baseline covariates will be included in models, including measures of preinjury functioning obtained at the acute assessment (eg, retrospective ratings of preinjury symptoms, history of migraine headaches) and demographic characteristics potentially related to outcomes (eg, age at injury, socioeconomic status, race/ethnicity and sex).

\section{Statistical power}

The proposed sample size of 700 in the mTBI group and 300 in the OI group provides adequate power for the study. Mixed-effects models are a generalisation of multiple regression models. In a multiple regression setting, with a sample size of 800 (allowing for 20\% attrition), p of 0.05 and 20 predictors, we would have statistical power of $80 \%$ to detect an effect size of $\mathrm{f}^{2}=0.01$ for the change in $\mathrm{R}^{2}$ attributable to any single predictor. This corresponds approximately to a change in $\mathrm{R}^{2}=0.01$ with a total $R^{2}=0.20$ for the overall model. Because individual genes and SNPs tend to, individually, account for very little variation (around 1\%), we powered the study to detect modest differences such as these. As this is anticipated to have a relatively small influence on the variance, we anticipate that for all other independent variables, power will be much greater than $80 \%$.

\section{POTENTIAL LIMITATIONS}

The study has several potential limitations. First, not all children with mTBI present to paediatric ED; thus, the study's findings may not be generalisable to children presenting to other settings or who do not seek medical care. Second, although the study is designed to assess the diagnostic and prognostic significance of a broad pool of neurobiological and psychosocial markers, some potential markers were not included. For instance, we did not include specific measures of depression, anxiety or somatisation because we preferred measures that were briefer and less burdensome, given the variety of psychosocial constructs we wished to assess; thus, we chose the Strengths and Difficulties Questionnaire, which is a standardised and widely used questionnaire that assesses emotional problems and other aspects of psychosocial adjustment. Lastly, the study does not include detailed measures of vestibular-ocular or cervicogenic problems. Those outcomes were not the focus of the study. Moreover, they are difficult to measure accurately without time-consuming clinical assessments, and we did not want to introduce additional burden to the participants. The study will only assess vestibular-ocular and cervicogenic problems indirectly, based on ratings of relevant postconcussive symptoms and pain, as well as assessment of balance.

\section{ETHICS AND DISSEMINATION}

The study received ethics approval from the ethics boards of all participating institutions (Conjoint Health Research Ethics Board at the University of Calgary, REB152296; Ste Justine Research Institute, University of Montreal, MP-21-2017-1332; CHEO REB16/23E; University of Alberta HREB, 64780; University of British Columbia Children's \& Women's Research Ethics Board, H16-00104). No significant risks are associated with participation in the study, and participation does not affect the care provided to participants. MRI is not associated 
with any known physical risks as long as participants are screened appropriately for MRI contraindications. The genes to be studied may affect recovery from injury but are not proven to do so. None of the genes are linked to a definitive health risk, and therefore genetic analyses will not uncover additional health information that has any significance. As such, we do not release any information about genetic analyses to participants. Psychological risks are limited to any discomfort that participants experience while completing study procedures, including MRI. Parents and children are approached in a sensitive and caring way to minimise any psychological or emotional discomfort, and research assistants stop any procedures if children or parents experience significant discomfort. Participants receive no direct benefit from being in the study but receive small incentives for completing study visits and remote ratings. Their participation may indirectly facilitate their recovery, but there is no guarantee that the research will help them. Parents receive a brief written summary of their children's test results. They are provided with clinical referrals if indicated or requested.

Research personnel take all appropriate and customary steps to ensure that data remain secure and that patient privacy and confidentiality is maintained. All members of the research team sign a confidentiality agreement. Only members of the research team are granted access to the REDCap database or CIPAC MRI database, which are password protected. Users are assigned access rights to databases in accordance with study responsibilities to ensure they have only the minimum required rights to perform their duties. All identifying information that is collected is flagged in the database and removed from data export unless the identifying information is required for statistical analysis. Contact information for participants is collected on a discrete instrument. Research staff at each site have rights to input the contact information in REDCap but not to export this information. Only the coordinator at each site has rights to both view and export contact information. Data access and entry are fully audited.

The study relies on an integrated knowledge translation plan that responds directly to the concerns of children, parents, healthcare providers, institutional decision-makers and other knowledge users. Central elements of the plan include an advisory committee to provide ongoing stakeholder participation, patient engagement researchers to assure a patient-centred focus, and a dedicated knowledge broker who will facilitate the development of a broad translational network and build a strategy for the dissemination and uptake of study findings by both scientists and end users. ${ }^{75} 76$ The knowledge broker will build on established national and international collaborations and partnerships between the study investigators and relevant professional societies, governmental and non-governmental organisations, policy-makers, healthcare administrators and providers, community groups and other end users. Scientific results will be disseminated at regional, national and international conferences and in manuscripts in peer-reviewed journals.

\section{Significance}

The study will improve the clinical care and outcomes of children with mTBI. Its findings will identify useful diagnostic and prognostic markers and clarify how they work in concert, enabling clinicians to better diagnose children with mTBI and target interventions to those at risk for poor outcomes. The knowledge translation plan will purposively drive change in clinical care by disseminating study findings to end users to help reduce practice variation and thereby promote better outcomes for children with $\mathrm{mTBI}$.

\section{Author affiliations}

${ }^{1}$ Department of Psychology, University of Calgary, Calgary, Alberta, Canada ${ }^{2}$ Hotchkiss Brain Institute, University of Calgary, Calgary, Alberta, Canada ${ }^{3}$ Alberta Children's Hospital Research Institute, University of Calgary, Calgary, Alberta, Canada

${ }^{4}$ Department of Psychology, Universite de Montreal and Ste Justine Hospital, Montreal, Québec, Canada

${ }^{5}$ Department of Pediatrics, University of Alberta and Stollery Children's Hospital,

Edmonton, Alberta, Canada

${ }^{6}$ Department of Pediatrics, University of British Columbia and BC Children's Hospital, Vancouver, British Columbia, Canada

${ }^{7}$ Department of Pediatrics and Emergency Medicine, Children's Hospital of Eastern Ontario, University of Ottawa, Ottawa, Ontario, Canada

${ }^{8}$ Department of Pediatrics, Universite de Montreal and Ste Justine Hospital,

Montreal, Québec, Canada

${ }^{9}$ Department of Radiology, University of Calgary, Calgary, Alberta, Canada

${ }^{10}$ Department of Radiology, University of Ottawa and Children's Hospital of Eastern

Ontario, Ottawa, Ontario, Canada

${ }^{11}$ Department of Biomedical Engineering, University of Alberta, Edmonton, Alberta, Canada

${ }^{12}$ Department of Radiology, Radiooncology and Nuclear Medicine, Université de

Montréal and Ste Justine Hospital, Montreal, Québec, Canada

${ }^{13}$ Department of Medical Genetics, University of Calgary and Alberta Children's Hospital, Calgary, Alberta, Canada

${ }^{14}$ Department of Community Health Sciences, University of Calgary, Calgary, Alberta, Canada

${ }^{15}$ Department of Clinical Neurosciences, University of Calgary, Calgary, Alberta, Canada

${ }^{16}$ Department of Pediatrics, University of Calgary and Alberta Children's Hospital, Calgary, Alberta, Canada

${ }^{17}$ Department of Medical Genetics, University of Calgary and Alberta Children's Hospital, Calgary, Alberta, Canada

${ }^{18}$ Faculty of Kinesiology, University of Calgary, Calgary, Alberta, Canada

${ }^{19}$ Research Innovation and Analytics, Alberta Health Services, Calgary, Alberta, Canada

Correction notice This article has been corrected since it was published, to improve readability and correct punctuation.

Acknowledgements The authors want to thank all current and future participants and their families, as well as ED staff, for their support of the study. The authors also want to thank the research coordinators, research assistants and other staff involved in the conduct of the study (in alphabetical order): Laurie Argourd, Christine Bon, Yvonne Brandelli, Ramona Cook, Lauren Dawson, Jessie Dhillon, Kevin Fitzpatrick, Maryse Lagace, Lisa Marie Langevin, Marc Lebel, Candice McGahern, Karen Pulsifer, Tina Samuel, Emily Schorr, Peter Seres, Barry Smith and Maria Tran.

Contributors KY, MB, WC, QD and RZ designed the study with input from all other authors. BB, BG, NA, CB, MD, SD, AH and CL guided the development of the neuroimaging protocol. $\mathrm{FB}$ and $\mathrm{RL}$ planned the genetic component of the study. TW and $\mathrm{KY}$ developed the biostatistical plan. $\mathrm{KM}$ and $\mathrm{KY}$ designed the knowledge translation plan. KY wrote the first draft of the manuscript. All authors read, critically revised and approved the final version of the manuscript. 
Funding This work is supported by a Canadian Institute of Health Research (CIHR) Foundation Grant (FDN143304) to KY, as well as by funds from the Alberta Children's Hospital Foundation and Alberta Children's Hospital Research Institute.

\section{Competing interests None declared.}

Ethics approval Conjoint Health Research Ethics Board at the University of Calgary. Provenance and peer review Not commissioned; externally peer reviewed. Open Access This is an Open Access article distributed in accordance with the Creative Commons Attribution Non Commercial (CC BY-NC 4.0) license, which permits others to distribute, remix, adapt, build upon this work non-commercially, and license their derivative works on different terms, provided the original work is properly cited and the use is non-commercial. See: http://creativecommons.org/ licenses/by-nc/4.0/

(C) Article author(s) (or their employer(s) unless otherwise stated in the text of the article) 2017. All rights reserved. No commercial use is permitted unless otherwise expressly granted.

\section{REFERENCES}

1. Faul M, Xu L, Wald MM, et al; Traumatic brain injury in the United States: emergency department visits, hospitalizations and deaths 2002-2006. Atlanta, GA: Centers for Disease Control and Prevention, National Center for Injury Prevention and Control, 2010.

2. Gilchrist J, Thomas KE, Xu L, et al. Nonfatal traumatic brain injuries related to sports and recreation activities among persons aged $\leq 19$ years--United States, 2001-2009. MMWR Morb Mortal Wkly Rep 2011;60:1337-42.

3. Barlow KM. Postconcussion syndrome: a review. J Child Neurol 2016;31:57-67.

4. Novak Z, Aglipay M, Barrowman N, et al. Association of Persistent Postconcussion Symptoms with Pediatric Quality of Life. JAMA Pediatr 2016;170:e162900.

5. Carroll LJ, Cassidy JD, Holm L, et al. Methodological issues and research recommendations for mild traumatic brain injury: the WHO collaborating Centre Task Force on Mild traumatic brain Injury. $J$ Rehab Med, Supp/2004;43:113-25.

6. Centers for Disease Control and Prevention (CDC), National Center for Injury Prevention and Control. Report to Congress on mild traumatic brain injury in the United States: steps to prevent a serious public health problem. Atlanta, GA: Centers for Disease Control and Prevention, 2003.

7. NIH Consensus Panel on Rehabilitation of Persons with Traumatic Brain Injury. Rehabilitation of persons with traumatic brain injury. JAMA 1999;282:974-83.

8. Register-Mihalik JK, Guskiewicz KM, Mihalik JP, et al. Reliable change, sensitivity, and specificity of a multidimensional concussion assessment battery: implications for caution in clinical practice. $J$ Head Trauma Rehabil 2013;28:274-83.

9. Zemek R, Barrowman N, Freedman SB, et al. Clinical risk score for persistent postconcussion symptoms among children with acute concussion in the ED. JAMA2016;315:1014-25.

10. McNally KA, Bangert $B$, Dietrich $A$, et al. Injury versus noninjury factors as predictors of postconcussive symptoms following mild traumatic brain injury in children. Neuropsychology 2013;27:1-12.

11. McCrea M, Broshek DK, Barth JT. Sports concussion assessment and management: future research directions. Brain Inj 2015;29:276-82.

12. Babcock L, Byczkowski T, Wade SL, et al. Predicting postconcussion syndrome after mild traumatic brain injury in children and adolescents who present to the emergency department. JAMA Pediatr 2013;167:156-61.

13. Zemek R, Osmond MH, Barrowman N; Pediatric Emergency Research Canada (PERC) Concussion Team. Predicting and preventing postconcussive problems in paediatrics $(5 \mathrm{P})$ study: protocol for a prospective multicentre clinical prediction rule derivation study in children with concussion. BMJ Open2013;3:e003550.

14. McAllister TW. Genetic factors modulating outcome after neurotrauma. PM\&R 2010;2:S241-S252.

15. Bonnano GA. Loss, trauma, and human resilience: have we underestimated the human capacity to thrive after extremely aversive events? Amer Psychol 2004;59:20-8.

16. Anderson MB, Williams JM. A model of stress and athletic injury: prediction and prevention. J Sport \& Exer Physio 1988;10:294-306.

17. Yeates KO, Taylor HG, Rusin J, et al. Longitudinal trajectories of postconcussive symptoms in children with mild traumatic brain injuries and their relationship to acute clinical status. Pediatrics 2009;123:735-43.

18. McCauley SR, Wilde EA, Anderson VA, et al. Recommendations for the use of common outcome measures in pediatric traumatic brain injury research. J Neurotrauma 2012;29:678-705.

19. American Association for Automotive Medicine. The Abbreviated Injury Scale (AIS)-1990 Revision. Des Plaines: American Association for Automotive Medicine, 1990.

20. Yeates KO, Kaizar E, Rusin J, et al. Reliable change in postconcussive symptoms and its functional consequences among children with mild traumatic brain injury. Arch Pediatr Adolesc Med 2012;166:585-684

21. Taylor HG, Dietrich A, Nuss K, et al. Post-concussive symptoms in children with mild traumatic brain injury. Neuropsychology 2010;24:148-59.

22. Dunning J, Batchelor J, Stratford-Smith $P$, et al. A meta-analysis of variables that predict significant intracranial injury in minor head trauma. Arch Dis Childhood2004;89:653-9.

23. Dunning J, Daly JP, Lomas JP, et al. Derivation of the children's head injury algorithm for the prediction of important clinical events decision rule for head injury in children. Arch Dis Child 2006;91:885-91.

24. Kuppermann N, Holmes JF, Dayan PS, et al. Identification of children at very low risk of clinically-important brain injuries after head trauma: a prospective cohort study. Lancet2009;374:1160-70.

25. Gorelick MH, Atabake SM, Hoyle J, et al. Interobserver agreement in assessment of clinical variables in children with blunt head trauma. Acad Emerg Med2008;15:812-8.

26. Moran LM, Taylor HG, Ganesalingam K, et al. Apolipoprotein E4 as a predictor of outcomes in pediatric mild traumatic brain injury. $J$ Neurotrauma,... 2009;26:1489-95.

27. Guskiewicz KM. Postural stability assessment following concussion: one piece of the puzzle. Clin J Sport Med 2001;11:182-9.

28. Guskiewicz KM, Ross SE, Marshall SW. Postural stability and neuropsychological deficits after concussion in collegiate Athletes. $J$ Ath Training 2001;36:263-73.

29. Riemann BL, Guskiewicz K, Shields EW. Relationship between clinical and forceplate measures of postural stability. J Sport Rehab 1999;8:71-82.

30. McLeod TCV, Barr WB, McCrea M, et al. Psychometric and measurement properties of concussion assessment tools in youth sports. J Ath Training 2006;41:399-408.

31. Khetani AM, Brooks BL, Mikrogianakis A, et al. Incorporating a computerized cognitive battery into emergency department care of pediatric mild traumatic brain injuries-Is it feasible? Ped Emerg Care. In press.

32. Brooks BL, Khan S, Daya H, et al. Neurocognition in the Emergency Department after a mild traumatic brain injury in youth. $J$ Neurotrauma 2014;31:1744-9.

33. Brooks BL, Daya $\mathrm{H}$, Khan $\mathrm{S}$, et al. Cognition in the emergency department as a predictor of recovery after pediatric mild traumatic brain injury. J Int Neuro Soc 2016;22:379-87.

34. Brooks BL, Low TA, Daya $\mathrm{H}$, et al. Test or rest? Computerized cognitive testing in the emergency department after pediatric mild traumatic brain injury does not delay symptom recovery. $J$ Neurotrauma 2016;33:2091-6.

35. Carskadon MA, Acebo C. A self-administered rating scale for pubertal development. J Adolesc Health 1993;14:190-5.

36. Ebesutani C, Drescher CF, Reise SP, et al. The loneliness questionnaire-short version: an evaluation of reverse-worded and non-reverse-worded items via item response theory. J Pers Assess 2012;94:427-37.

37. Asher SR, Hymel S, Renshaw PD. Loneliness in children. Child Development 1984:1456-64.

38. Kerres Malecki C, Kilpatrick Demary M. Measuring perceived social support: development of the child and adolescent social support scale (CASSS). Psychology in the Schools 2002;39:1-18.

39. Hewitt PL, Caelian CF, Flett GL, et al. Perfectionism in children: associations with depression, anxiety, and anger. Personality and Individual Differences 2002;32:1049-61.

40. Connor KM, Davidson JR. Development of a new resilience scale: the Connor-Davidson Resilience Scale (CD-RISC). Depress Anxiety 2003;18:76-82.

41. Lukow HR, Godwin EE, Marwitz JH, et al. Relationship between resilience, adjustment, and functioning after traumatic brain injury: a preliminary report. J Head Trauma Rehabil 2015;30:241-8.

42. Allen JL, Rapee RM, Sandberg S. Assessment of maternally reported life events in children and adolescents: a comparison of interview and checklist methods. J Psychopathol Behav Assess 2012;34:204-15. 
43. Haug E, Rasmussen M, Samdal O, et al. Overweight in school-aged children and its relationship with demographic and lifestyle factors: results from the WHO-Collaborative Health Behaviour in School-aged Children (HBSC) study. Int J Public Health 2009;54(Suppl 2):167-79.

44. LeBourgeois MK, Giannotti F, Cortesi F, et al. The relationship between reported sleep quality and sleep hygiene in Italian and American adolescents. Pediatrics2005;115:257-65.

45. Byles J, Byrne C, Boyle $\mathrm{MH}$, et al. Ontario child health study: reliability and validity of the general functioning scale of the McMaster family assessment device. Family Process 1988;27:97-104.

46. Speranza M, Guénolé F, Revah-Levy A, et al. The French version of the Family Assessment Device. Can J Psychiatry 2012;57:570-7.

47. Yeates KO, Taylor HG, Rusin J, et al. Premorbid child and family functioning as predictors of post-concussive symptoms in children with mild traumatic brain injuries. Int J Dev Neurosci 2012;30:231-7.

48. Noel M, Palermo TM, Essner B, et al. A developmental analysis of the factorial validity of the parent-report version of the adult responses to Children's Symptoms in children versus adolescents with chronic pain or pain-related chronic illness. J Pain 2015;16:31-41.

49. Kessler RC, Barker PR, Colpe LJ, et al. Screening for serious mental illness in the general population. Arch Gen Psychiatry 2003;60:184-9.

50. Kessler RC, Green JG, Gruber MJ, et al. Screening for serious mental illness in the general population with the $\mathrm{K} 6$ screening scale: results from the WHO World Mental Health (WMH) survey initiative. Int $J$ Methods Psychiatr Res 2010;19(Suppl 1):4-22.

51. Moriarty DG, Zack MM, Kobau R. The Centers for Disease Control and Prevention's Healthy Days Measures - population tracking of perceived physical and mental health over time. Health Qual Life Outcomes 2003;1:37-8.

52. Ayr LK, Yeates KO, Taylor HG, et al. Dimensions of postconcussive symptoms in children with mild traumatic brain injuries. J Int Neuropsychol Soc 2009;15:19-30.

53. Mittenberg W, Wittner MS, Miller LJ. Postconcussion syndrome occurs in children. Neuropsychology 1997;11:447-52.

54. Moran LM, Taylor HG, Rusin J, et al. Do postconcussive symptoms discriminate injury severity in pediatric mild traumatic brain injury? $J$ Head Trauma Rehabil 2011;26:348-54.

55. Kosinski M, Bayliss MS, Bjorner JB, et al. A six-item short-form survey for measuring headache impact: the HIT-6. Qual Life 2003;12:963-74.

56. Becker SP, Ramsey RR, Byars KC. Convergent validity of the Child Behaviour Checklist sleep items with validated sleep measures and sleep disorder diagnoses in children and adolescents referred to a sleep disorders center. Sleep Medicine 2015;16:79-86.

57. Claar RL, Walker LS. Functional assessment of pediatric pain patients: psychometric properties of the functional disability inventory. Pain 2006;121:77-84.

58. Walker LS, Greene JW. The functional disability inventory: measuring a neglected dimension of child health status. J Pediatr Psychol 1991;16:39-58.

59. Ewing-Cobbs L, Bloom DR, Prasad MR, et al. Assessing recovery and disability after physical trauma: the Pediatric Injury Functional Outcome Scale. J Pediatr Psychol 2014;39:653-65.
60. Varni JW, Limbers CA. The pediatric quality of life inventory: measuring pediatric health-related quality of life from the perspective of children and their parents. Pediatr Clin North Am 2009;56:843-63.

61. Varni JW, Limbers CA, Burwinkle TM. Parent proxy-report of their children's health-related quality of life: An analysis of 13,878 parents' reliability and validity across age subgroups using the PedsQL ${ }^{\mathrm{TM}} 4.0$ Generic Core Scales. Health Qual Life Outcomes 2007;5:1-10.

62. Beers SR, Wisniewski SR, Garcia-Filion P, et al. Validity of a pediatric version of the Glasgow Outcome Scale-Extended. J Neurotrauma 2012;29:1126-39.

63. Chevignard M, Kerrouche B, Lind K, et al. Validation of the French translation of the Glasgow Outcome Scale-Extended, Pediatric version (GOS-E Peds): Clinical utility in assessing outcome in children and adolescents following acquired brain injury (ABI). Ann Phys Rehabil Med 2015;58:e138.

64. Goodman R. The extended version of the Strengths and Difficulties Questionnaire as a guide to child psychiatric caseness and consequent burden. J Child Psychol Psychiatry 1999;40:791-9.

65. The Psychological Corporation. Wechsler Abbreviated Scale of Intelligence. San Antonio: The Psychological Corporation, 1999

66. von Baeyer CL, Spagrud LJ, McCormick JC, et al. Three new datasets supporting use of the Numerical Rating Scale (NRS-11) for children's self-reports of pain intensity. Pain 2009;143:223-7.

67. Reynolds CR, Kamphaus RW. Behaviour Assessment System for Children-. Second Edition. Circle Pines, MN: AGS Publishing, 2004.

68. Green P. Manual for Green's Medical Symptom Validity Test (MSVT). Edmonton: Green's Publishing Inc, 2004.

69. Kirkwood MW, Kirk JW. The base rate of suboptimal effort in a pediatric mild TBI sample: performance on the medical symptom validity test. Clin Neuropsychol 2010;24:860-72.

70. Kirkwood MW, Yeates KO, Randolph C, et al. The implications of symptom validity test failure for ability-based test performance in a pediatric sample. Psychological assessment 2012;24:36-45.

71. Harris P, Taylor R, Thielke R, et al. Research electronic data capture (REDCap): A metadata-driven methodology and workflow process for providing translational research informatics support. J Biomedical Infor 2009;42:377-81.

72. Sinharay S, Stern HS, Russell D. The use of multiple imputation for the analysis of missing data. Psychological Methods 2001;6:317-29.

73. Burchinal MR, Bailey DB, Snyder P. Using Growth Curve Analysis to Evaluate Child Change in Longitudinal Investigations. J Early Interv 1994;18:403-23.

74. Hu FB, Goldberg J, Hedeker D, et al. Comparison of populationaveraged and subject-specific approaches for analyzing repeated binary outcomes. Am J Epidemiol 1998;147:694-703.

75. Bornbaum CC, Kornas K, Peirson L, et al. Exploring the function and effectiveness of knowledge brokers as facilitators of knowledge translation in health-related settings: a systematic review and thematic analysis. Implementation Science 2015;10:162.

76. Canadian Health Services Research Foundation. The theory and practice of knowledge brokering in Canada's health system. 2003 www.cfhi-fcass.ca/migrated/pdf/theory_and_practice_e.pdf. 